\title{
Efectos del consumo de café sobre la conducta alimentaria en ratas*
}

\section{Effects of coffee intake on feeding behavior in rats}

\author{
Virginia Aguilera \\ Antonio López-Espinoza** \\ Resumen \\ Alma Gabriela Martínez \\ Felipe Díaz \\ Elia Valdez \\ Alma Galindo \\ Carolina De la Torre-Ibarra \\ Centro de Investigaciones en \\ Comportamiento Alimentario \\ y Nutrición (CICAN) Cusur - \\ Universidad de Guadalajara, México \\ Recibido: 23 de agosto de 2008 \\ Revisado: 4 de octubre de 2008 \\ Aceptado: 10 de noviembre de 2008

\begin{abstract}
El objetivo del presente estudio fue evaluar los efectos del consumo de café con y sin cafeína sobre la conducta alimentaria en ratas. Dieciséis ratas fueron expuestas a 2, 3, 4 y 5 gramos de café con cafeína y sin cafeína diluidas en $200 \mathrm{ml}$ de agua. Los sujetos fueron asignados a cuatro grupos conformados por dos hembras y dos machos cada uno. Los grupos 1 y 2 fueron expuestos a café con cafeína y los grupos 3 y 4 a café sin cafeína. La exposición de café para los grupos 1 y 3 fue de forma ascendente $(2 \mathrm{~g}, 3 \mathrm{~g}, 4 \mathrm{~g}$ y $5 \mathrm{~g})$ y para los grupos 2 y 4 de forma descendente $(5 \mathrm{~g}, 4$ $\mathrm{g}, 3 \mathrm{~g}$ y $2 \mathrm{~g}$ ). El experimento fue dividido en nueve fases. Las fases 1, 3, 5, 7 y 9 tuvieron una duración de diez días, en cada una se proporcionaron $200 \mathrm{ml}$ de agua y $50 \mathrm{~g}$ de alimento. Las fases 2, 4, 6 y 8 tuvieron una duración de cinco días cada una, en las cuales se les proporcionó las concentraciones de café y $50 \mathrm{~g}$ de alimento. Los resultados mostraron que las concentraciones bajas de café registraron un mayor consumo en comparación con las concentraciones altas en ambas condiciones (ascendente y descendente). Por otra parte, el café sin cafeína fue más consumido que el café con cafeína.
\end{abstract} \\ Palabras clave: rata, café, consumo, conducta ali- \\ mentaria. \\ * Esta investigación fue financiada por el proyecto 46083-H del CONACyT. \\ ** Correspondencia: Dr. Antonio López-Espinoza, Centro de Investigaciones en Comportamiento Alimentario y Nutrición, Centro Universitario del Sur, Uni- \\ versidad de Guadalajara, Prolongación Colón s/n, Edificio X-3, Zapotlán el Grande, Jalisco, México. Web: www.cusur.udg.mx/cican/ Correos electróni- \\ cos: Virginia Aguilera: virginia.aguilera@cusur.udg.mx; Antonio López -Espinoza: antonio.lopez@cusur.udg.mx
}




\section{Abstract}

The objective of the present study was to evaluate the effects of coffee intake with and without caffeine on feeding behavior in rats. Sixteen rats was exposed to $2 \mathrm{~g}, 3 \mathrm{~g}, 4 \mathrm{~g}$ and $5 \mathrm{~g}$ of coffee with caffeine and without caffeine diluted in $200 \mathrm{ml}$ of water. Subjects were assigned to 4 groups conformed by 2 females and 2 males each one. Groups 1 and 2 were exposed to coffee with caffeine and groups 3 and 4 to coffee without caffeine. Exposed to coffee for groups 1 and 3 was of ascending form ( $2 \mathrm{~g}, 3 \mathrm{~g}, 4 \mathrm{~g}$, and $5 \mathrm{~g})$ and for groups 2 and 4 of descendent form $(5 \mathrm{~g}, 4 \mathrm{~g}, 3 \mathrm{~g}$ and $2 \mathrm{~g})$. The experiment was divided in 9 phases. Phases 1, 3, 5, 7 and 9 lasted of 10 days, in each one were provided $200 \mathrm{ml}$ of water and $50 \mathrm{~g}$ food. Phases 2, 4, 6 and 8 lasted of 5 days; in each one provided the concentrations of coffee and $50 \mathrm{~g}$ food. The results showed that the low concentrations of coffee registered a greater consumption in comparison with the high concentrations in both conditions (ascending and descendent). On the other hand, coffee without caffeine was more consumed than coffee with caffeine.

Key words: rats, coffee, intake, feeding behavior.

\section{Introducción}

La evidencia experimental demuestra que diversos factores determinan la selección y consumo de los alimentos. Entre los más importantes se encuentran:

- El sabor, el cual, a su vez, está determinado por las características químicas del alimento y las experiencias alimentarias de los organismos a edades tempranas. Adicionalmente, se ha documentado que existe una preferencia por los sabores dulces en comparación con los amargos (Rozin, 2002).

- Las consecuencias posingestivas.

- El contexto alimentario.

- El contenido nutricio (Bennett \& Galef, 1981; Sclafani, 1990).

Una particularidad del sabor amargo es que se considera como no agradable; sin embargo, al ser mezclado con endulzantes su preferencia cambia y los organismos lo consumen, un ejemplo es el café (Capaldi, 1996). Este tipo de bebida tiene un alto consumo en todo el mundo, en promedio las personas consumen de dos a tres tazas de café por día (James, 1997). Algunas de las motivaciones para consumir café involucran propiedades como su sabor, olor, efectos positivos de la cafeína, la aversión al síndrome de la abstinencia de cafeína, la interacción social y el deseo de ingerir una sustancia baja en calorías (Rozin, 2002).

Se considera que la conducta de consumir café se incrementa por ser una bebida estimulante debido a su contenido de cafeína (Chou, 1992; Prasad, 1998; Messer, 2002). La cafeína es un alcaloide de la familia de las xantinas, su absorción y distribución en los organismos se lleva a cabo a través de los tejidos, actúa como relajante y aumenta la actividad del córtex cerebral (Cuadrado-Calleja, 2003; Stafford, 2003; Uraga, Guijarro, Pozas \& Blanco, 2002).

La cafeína al ser absorbida y metabolizada por el organismo afecta el sistema nervioso central y se manifiesta en un aumento de la actividad general, la memoria, la atención, la concentración y el aprendizaje, entre otras (Tieges, Ridderinkhof, Snel, \& Kok, 2004). Por otra parte, es importante mencionar que a la cafeína se le han atribuido efectos dañinos como: adicción, insomnio, taquicardia, ansiedad, así como su relación con enfermedades coronarias y cáncer (Chou, 1992; Cuadrado-Calleja, 2003; Stafford, 2003).

Evidencia experimental demuestra que el apareamiento de un sabor con la cafeína provoca un aumento en su preferencia y consumo debido a sus consecuencias posingestivas (Yeomans, et al., 2000; Griffiths, Bigelow \& Liebson, 1989). La cafeína al ser consumida en bajas dosis en el 
café, refrescos u otros alimentos provoca efectos positivos en el organismo y garantiza su consumo (Rozin, 2002; Tieges et al., 2004). Por otro lado, cuando es consumida en altas dosis provoca efectos negativos y grados de toxicidad que llevan a disminuir su ingesta (Cuadrado-Calleja, 2003).

El principal interés para el desarrollo de este estudio es el análisis del patrón de consumo en ratas, expuestas a diferentes concentraciones de café, ya que consideramos que en este tipo de conducta interactúan elementos como: sabor, preferencia, consecuencias posingestivas, disposición del alimento, contenido nutricio y la historia alimentaria (Bennett \& Galef, 1981; Capaldi, 1996: Rozin, 2002; López-Espinoza, 2004).

El objetivo de utilizar café con cafeína y café sin cafeína en el presente estudio fue evaluar las posibles diferencias en su ingesta, relacionadas con las consecuencias posingestivas de la cafeína y las diferentes concentraciones de café utilizadas. Por tal motivo, se utilizó un análisis paramétrico en las concentraciones de café.

\section{Método}

\section{Sujetos}

Dieciséis ratas albinas de tres meses de edad, ocho machos (M1, M2, M3, M4, M5, M6, M7, M8) y ocho hembras $(\mathrm{H} 1, \mathrm{H} 2, \mathrm{H} 3, \mathrm{H} 4, \mathrm{H} 5, \mathrm{H} 6, \mathrm{H} 7, \mathrm{H} 8)$ e ingenuas experimentalmente.

\section{Aparatos y materiales}

Se emplearon dieciséis cajas habitación para ratas con medidas de: $13 \mathrm{~cm}$ de altura por $27 \mathrm{~cm}$ de ancho por $35 \mathrm{~cm}$ de largo, con una reja metálica en la parte superior con divisiones para comida y agua. En las cajas se puso una alfombra de aserrín, la cual se cambió por otra cada cinco días. Se utilizó una báscula para el registro del consumo de alimento y peso corporal de cada sujeto. Como alimento se utilizaron croquetas de la marca comercial Nutri-cubos que contienen los nutrientes estándares necesarios para animales de laborato- rio. Su fórmula nutricional es la siguiente: $3 \%$ de grasas, $7 \%$ de cenizas, $1 \%$ de calcio, $23 \%$ de proteína, $6 \%$ de fibra, $49 \%$ de E.L.N. (extracto libre de nitrógeno), $0,6 \%$ de fósforo y $12 \%$ de humedad. Como bebida se utilizó agua que fue administrada en bebederos graduados de $250 \mathrm{ml}$. Durante la manipulación experimental se utilizó café soluble en dosis de $2 \mathrm{~g}, 3 \mathrm{~g}, 4 \mathrm{~g}$ y $5 \mathrm{~g}$ de la marca comercial Great Value colombiano.

\section{Procedimiento}

Los sujetos experimentales se pusieron en cajas individuales, previamente identificados con un número de registro, fecha de nacimiento y peso corporal al inicio del experimento. El peso corporal, el consumo de agua, el consumo de alimento y el consumo de agua con café se registró diariamente a las 9:00 de la mañana. Para el pesaje se tomó la caja habitación y se trasladó a la mesa de trabajo en la que se encontraba la báscula utilizada para el registro. Se tomó a la rata y se introdujo en el recipiente de la báscula con lo que se registró el peso corporal. Al final de este procedimiento los sujetos retornaron a su caja habitación y permanecieron en el bioterio. El café soluble fue pesado de acuerdo con la dosis utilizada, posteriormente fue diluido en $200 \mathrm{ml}$ de agua. Las dosis de café se prepararon diariamente durante los días de exposición

\section{Diseño experimental}

Los sujetos fueron asignados de manera aleatoria a cuatro grupos conformados por dos hembras $y$ dos machos cada uno. Los grupos 1 y 2 fueron expuestos a café con cafeína, y los grupos 3 y 4 , a café sin cafeína. Las dosis de café utilizadas fueron de: 2 g, 3 g, 4 g y 5 g de café con cafeína y sin cafeína diluido en $200 \mathrm{ml}$ de agua. El grupo 1 fue conformado por los sujetos $M 1, M 2, \mathrm{H} 1$ y H2, el grupo 2 por los sujetos $\mathrm{M} 3, \mathrm{M} 4, \mathrm{H} 3$ y $\mathrm{H} 4$, el grupo 3 por los sujetos $\mathrm{M} 5, \mathrm{M} 6, \mathrm{H} 5$ y $\mathrm{H} 6$ y el grupo 4 por los sujetos M7, M8, H7 Y H8. Los grupos 1 y 3 fueron expuestos a las concentraciones de café de forma ascendente y los grupos 2 y 4 de forma descendente. El experimento fue dividido en nueve fases. 
Las fases 1, 3, 5, 7 y 9 tuvieron una duración de diez días cada una y se proporcionaron $200 \mathrm{ml}$ de agua y $50 \mathrm{~g}$ de alimento (libre acceso). Las fases 2 , 4, 6 y 8 tuvieron una duración de cinco días cada una, en las cuales se les proporcionó las dosis de café y $50 \mathrm{~g}$ de alimento. En la fase 2, a los grupos 1 y 3 se les proporcionó $2 \mathrm{~g}$ de café, y a los grupos
2 y 4 se les dio $5 \mathrm{~g}$. En la fase 4 , a los grupos 1 y 3 se les proporcionó $3 \mathrm{~g}$ de café, y a los grupos 2 y 4 se les dio $4 \mathrm{~g}$. En la fase 6 , a los grupos 1 y 3 se les proporcionó $4 \mathrm{~g}$ de café, y a los grupos 2 y 4 se les dio $3 \mathrm{~g}$. En la fase 8 , a los grupos 1 y 3 se les proporcionó $5 \mathrm{~g}$ de café, y a los grupos 2 y 4 se les dio $2 \mathrm{~g}$. Todas las dosis fueron diluidas en $200 \mathrm{ml}$ de agua. (tabla 1).

Tabla 1. Secuencia utilizada de exposición a las diferentes concentraciones de café con cafeína (c/c) y sin cafeína (s/c).

\begin{tabular}{lccccccccc}
\hline & Fase $\mathbf{1}$ & $\begin{array}{c}\text { Fase } \\
\mathbf{2}\end{array}$ & Fase $\mathbf{3}$ & $\begin{array}{c}\text { Fase } \\
\mathbf{4}\end{array}$ & Fase $\mathbf{5}$ & $\begin{array}{c}\text { Fase } \\
\mathbf{6}\end{array}$ & Fase $\mathbf{7}$ & $\begin{array}{c}\text { Fase } \\
\mathbf{8}\end{array}$ & Fase $\mathbf{9}$ \\
\hline $\begin{array}{l}\text { Grupos } \mathbf{1} \\
\text { c/c 3 s/c }\end{array}$ & $\begin{array}{c}\text { Libre } \\
\text { acceso }\end{array}$ & $\begin{array}{c}\mathbf{2} \mathrm{g} \\
\text { café }\end{array}$ & $\begin{array}{c}\text { Libre } \\
\text { acceso }\end{array}$ & $\begin{array}{c}3 \mathrm{~g} \\
\text { café }\end{array}$ & $\begin{array}{c}\text { Libre } \\
\text { acceso }\end{array}$ & $\begin{array}{c}4 \mathrm{~g} \\
\text { café }\end{array}$ & $\begin{array}{c}\text { Libre } \\
\text { acceso }\end{array}$ & $\begin{array}{c}5 \mathrm{~g} \\
\text { café }\end{array}$ & $\begin{array}{c}\text { Libre } \\
\text { acceso }\end{array}$ \\
$\begin{array}{l}\text { Grupos 2 } \\
\text { c/c 4 s/c }\end{array}$ & $\begin{array}{c}\text { Libre } \\
\text { acceso }\end{array}$ & $\begin{array}{c}5 \mathrm{~g} \\
\text { café }\end{array}$ & $\begin{array}{c}\text { Libre } \\
\text { acceso }\end{array}$ & $\begin{array}{c}4 \mathrm{~g} \\
\text { café }\end{array}$ & $\begin{array}{c}\text { Libre } \\
\text { acceso }\end{array}$ & $\begin{array}{c}3 \mathrm{~g} \\
\text { café }\end{array}$ & $\begin{array}{c}\text { Libre } \\
\text { acceso }\end{array}$ & $\begin{array}{c}2 \mathrm{~g} \\
\text { café }\end{array}$ & Libre \\
$\begin{array}{l}\text { Duracceso } \\
\text { (días) }\end{array}$ & 10 & 5 & 10 & 5 & 10 & 5 & 10 & 5 & 10 \\
\hline
\end{tabular}

\section{Resultados}

La figura 1 muestra los promedios del peso corporal y la figura 2 los promedios del consumo de alimento. La columna izquierda muestra los registros de las hembras y la derecha la de los machos. El panel superior muestra los datos de los grupos expuestos a café con cafeína y el panel inferior los grupos que fueron expuestos a café sin cafeína. La línea continua representa los periodos de libre acceso. Los círculos muestran el periodo de exposición a 2 g de café; los cuadros, a $3 \mathrm{~g}$; los rombos, a $4 \mathrm{~g}$; y los triángulos, a $5 \mathrm{~g}$ diluidos en $200 \mathrm{ml}$ de agua.

La figura 1 muestra los datos del peso corporal de hembras y machos que fueron expuestos a café con cafeína y sin cafeína. En general, todos los sujetos mostraron una curva de crecimiento propia de la especie. Los machos mostraron un peso corporal estable en comparación con las hembras en todos los grupos. De manera particular, las hembras del grupo 1 expuestas a café con cafeína de forma ascendente mostraron una disminución del peso corporal posterior a la exposición a 3 $\mathrm{g}$ de café (cuadros), que se recuperó de forma gradual durante la exposición a la concentración de $4 \mathrm{~g}$ (rombos), pero que disminuyó una vez más en la fase de $5 \mathrm{~g}$ (triángulos). En las hembras del grupo 2, que fueron expuestas al café con cafeína de manera descendente se observó una disminución del peso corporal durante la primera fase de café de $5 \mathrm{~g}$ (triángulos), el cual se recuperó en las siguientes fases, mostrando un aumento durante la exposición a $2 \mathrm{~g}$ de café. En las hembras y machos de los grupos 3 y 4 que fueron expuestos a café sin cafeína no se observaron cambios en el peso corporal. De manera particular, se observa que los machos del grupo 3 mostraron una disminución de su peso corporal durante la fase 4 ( $5 \mathrm{~g}$ de café), peso que fue recuperado en condiciones de libre acceso. 


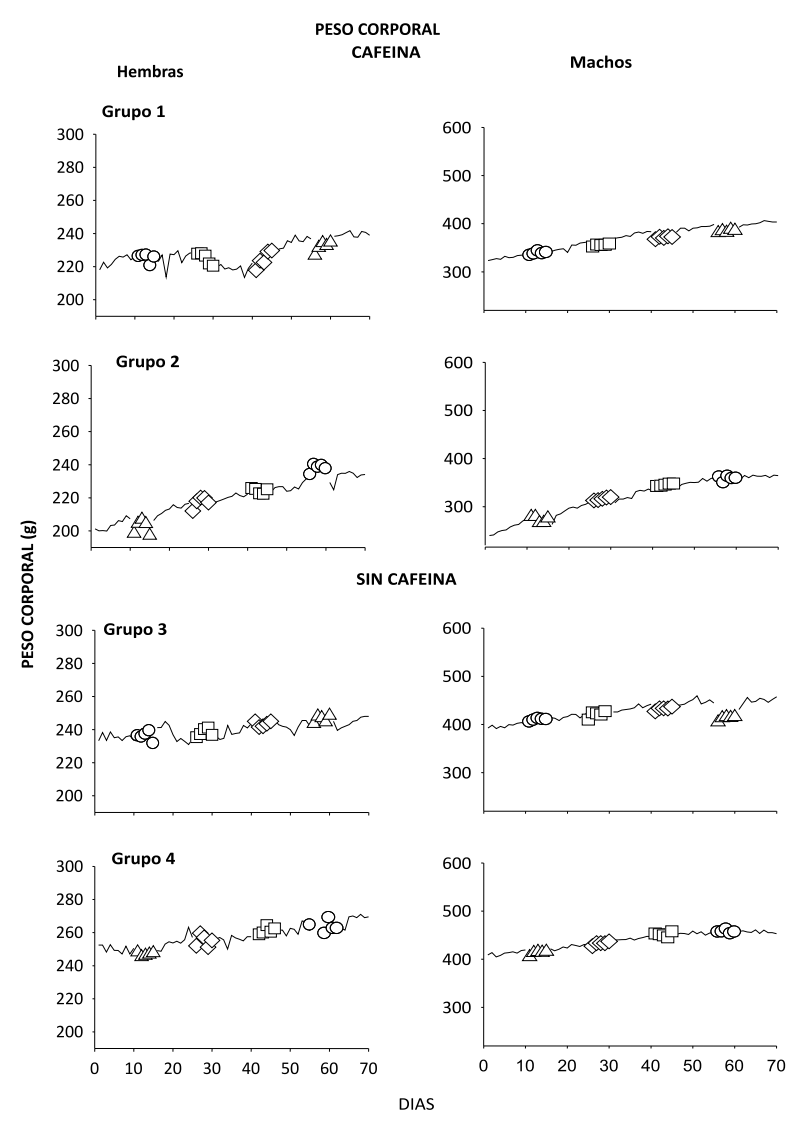

Figura 1. Muestra el promedio del peso corporal de las hembras y los machos que fueron expuestos a las concentraciones de café con cafeína y sin cafeína. La línea continua representa la condición de libre acceso. Las figuras representan los periodos de manipulación experimental. Los circulos representan la concentración de $2 \mathrm{~g}$ de café, los cuadros $3 \mathrm{~g}$, los rombos $4 \mathrm{~g}$ y los triángulos $5 \mathrm{~g}$

La figura 2 muestra el consumo de alimento de hembras y machos de los cuatro grupos. En general, se observa variabilidad en el consumo de alimento durante todo el experimento. Los sujetos disminuyeron su consumo durante los periodos de exposición a las concentraciones de café con cafeína y sin cafeína en todos los grupos. En el grupo 1 todos los sujetos mostraron disminuciones en el consumo de alimento en las fases posteriores a la exposición a $2 \mathrm{~g}$ de café. En el grupo 2 los machos mostraron una disminución durante su exposición a las dosis de $5 \mathrm{~g}$ y $4 \mathrm{~g}$ de café. En el grupo 3 los sujetos mostraron mayor variabilidad durante la exposición a 4 g y 5 g de café. En el grupo 4 los machos mostraron una disminución en el consumo de alimento durante la fase de $5 \mathrm{~g}$ de café.

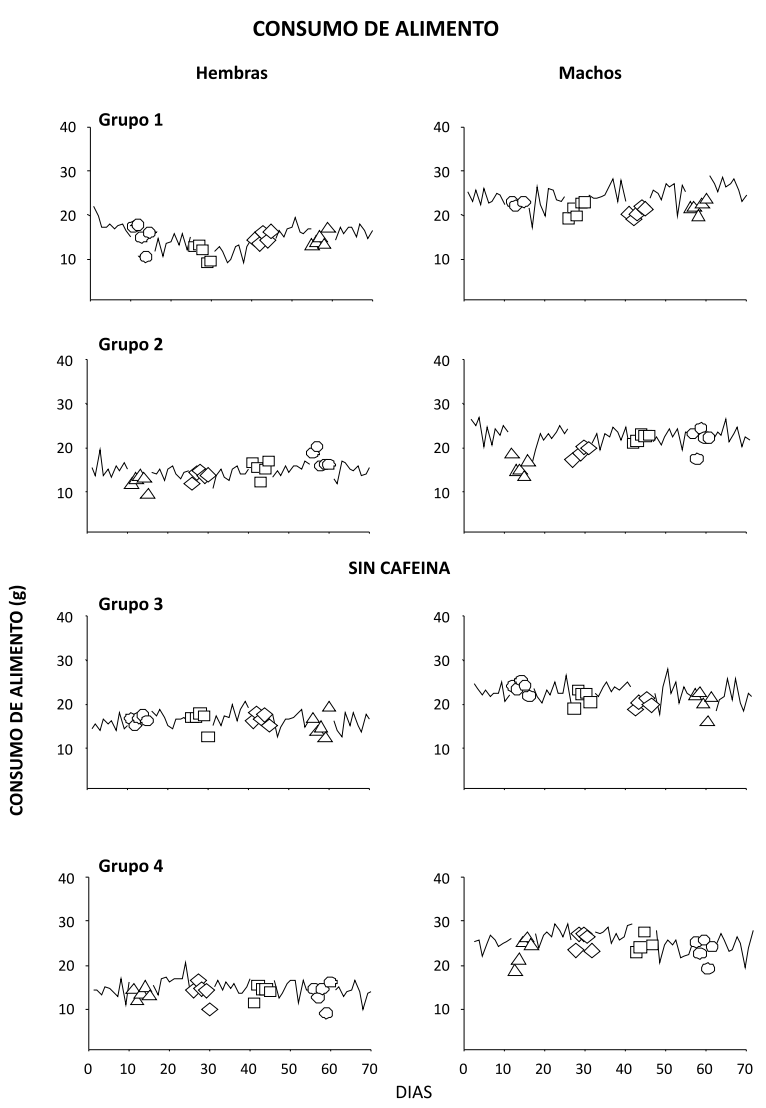

Figura 2. Muestra el promedio de consumo de alimento de hembras y machos que fueron expuestos a café con cafeína y sin cafeína. La línea continua representa la condición de libre acceso. Las figuras representan los periódos de manipulación experimental. Los círculos representan la concentración de $2 \mathrm{~g}$ de café, los cuadros $3 \mathrm{~g}$, los rombos $4 \mathrm{~g}$ y los triángulos $5 \mathrm{~g}$

La figura 3 muestra los promedios de consumo de café de hembras y machos de los cuatro grupos en las dos condiciones. La columna de la izquierda muestra los datos de las hembras y la derecha de los machos. En el panel superior se encuentran los grupos que fueron expuestos a la condición ascendente de café y en el panel inferior los grupos que fueron expuestos a la condición descendente de café. Las barras grises representan las dosis de café con cafeína y las barras blancas las de café 
sin cafeína. De manera general se observa que las dosis de café sin cafeína fueron más consumidas en comparación con las de café con cafeína. Ambos grupos muestran un mayor consumo de café con las dosis bajas y un menor consumo con las altas en ambas condiciones. Los consumos de café con cafeína en las hembras y machos de los grupos 1 y 3 disminuyeron conforme las dosis aumentaron $y$, por el contrario, para las hembras y machos de los grupos 2 y 4 los consumos de café con cafeína aumentaron conforme la dosis de café disminuía. Este mismo efecto se observa en las hembras y los machos expuestos a café sin cafeína de los grupos 2 y 4 . En cambio, en los grupos 1 y 3 de hembras y machos en condición ascendente no se observa este efecto.

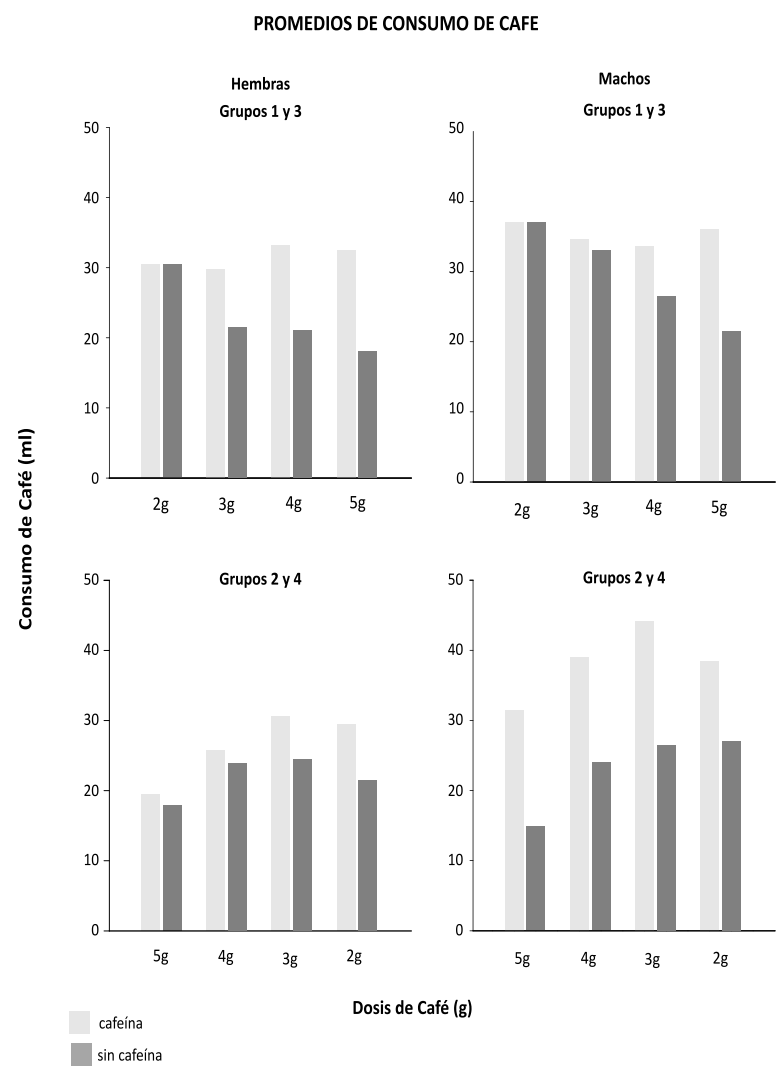

Figura 3. Muestra el consumo de café con o sin cafeína en las diferentes dosis utilizadas

\section{Discusión}

Los resultados obtenidos en el presente experimento muestran que el patrón de consumo de alimento fue modificado por la presencia de concentraciones de café diluidas en agua. Es relevante mencionar que el peso corporal de las hembras presentó modificaciones caracterizadas por disminuciones, debido a la exposición de las concentraciones altas de café. Por otro lado, el peso corporal de los machos no presentó cambios importantes.

Los cambios en el peso corporal pueden ser explicados por un fenómeno particular de regulación en los mamíferos que tienden a mantener constante su peso corporal, a pesar de posibles cambios en el balance energético y la disponibilidad de la comida. Es decir, cuando se pierde peso corporal existe una tendencia a retornar a los niveles anteriores a la pérdida. Con lo anterior es posible explicar por qué los animales presentan una tendencia a regular la ingesta de alimento para mantener el balance del peso corporal (Martínez, López-Espinoza \& Martínez, 2006).

Una particularidad del peso corporal es que varía de un organismo a otro e incluso entre las especies. Cuando a los animales se les permite comer libremente se observa una constante entre la ingesta de alimento y el gasto de energía. Los organismos que experimentan reducciones del peso corporal debido a las modificaciones en su dieta por procedimientos experimentales, regresan a un nivel adecuado para su edad y sexo después de eliminar dichos procedimientos (Martínez et al., 2006). Este fenómeno permite explicar los resultados obtenidos en el presente experimento, ya que bajo condiciones de manipulación experimental los sujetos presentaron una disminución de su peso corporal que posteriormente fue recuperado en condiciones de libre acceso. Lo anterior es observable en ambos grupos sin importar la secuencia en la manipulación experimental, ni a la condición a la que estuvieron expuestos.

El consumo de alimento se modificó en ambos grupos durante la presencia de las diferentes concentraciones de café. Lo anterior se explica debido a la mezcla utilizada (agua+café), ya que se presentó una variación de sabor, debido a la concentración utilizada (a mayor concentración de café, el sabor fue más intenso) lo que propi- 
ció una disminución del consumo de alimento con respecto a la línea base.

La evidencia experimental demuestra que los organismos mantienen un ajuste proporcional en relación con la disposición y el consumo de agua y alimento (Martínez \& López-Espinoza, 2007). Por tal motivo, en el presente estudio se observó una relación entre la cantidad de alimento consumido y el líquido consumido, caracterizado por un consumo proporcional de alimento-agua en condiciones de libre acceso y alimento-concentración de café, en las diferentes fases experimentales, manteniendo un patrón semejante de consumo en ambas condiciones. Es decir, a menor consumo de bebida menor ingesta de alimento.

Al respecto, Martínez y López-Espinoza (2007) señalaron que no sólo las señales internas de un organismo son las determinantes para que un alimento sea ingerido, sino que las variables externas como el olor o el sabor del alimento propician la conducta de comer. Mencionaron que las respuestas ante los estímulos pueden ser innatas o aprendidas como ocurre en la preferencia por ciertos sabores.

Los resultado obtenidos en el promedio de consumo de café probablemente se presentaron por los siguientes factores: el sabor amargo del café (Capaldi, 1996; Rozin, 2002); los efectos posingestivos de la cafeína, los cuales estuvieron determinados por la concentración de café en el agua (Sclafani, 1990; Bennett \& Galef, 1981); y el contenido no calórico de las soluciones de café (Fedorchak, Mesita, Plater \& Brougham, 2002).

De manera particular, el sabor amargo del café está relacionado con la evidencia experimental que demuestra que el sabor de los alimentos es determinante para que los organismos los consuman. En el caso de los humanos, el sabor es un factor determinante para la incorporación de nuevos alimentos a su dieta diaria. Sin embargo, aún existe discusión sobre si la asociación entre el sabor y la preferencia depende de la experiencia (Yeretzian, Pollien, Lindinger \& Ali, 2004; Messer, 2002; Rozin, 2002).
La evidencia experimental demuestra que las ratas prefieren aquellos sabores que son apareados con cafeína, los factores que facilitan esto son: las bajas concentraciones de cafeína y el estado de privación de los sujetos (Yeomans et al., 2000; Fedorchak et al., 2002) Los resultados del presente experimento demostraron que las concentraciones bajas de café $(2 \mathrm{~g})$ registraron un mayor consumo en comparación con las concentraciones altas.

El café es una bebida no calórica, no nutritiva y de sabor amargo. Debido a estas características se esperaría que fuera una bebida poco consumida. Al respecto Capaldi (1996) reportó que los sabores considerados como no agradables o no preferidos al ser mezclados con un sabor dulce como lo es el azúcar son altamente consumidos. Lo anterior se explica debido a que existe una fuente alterna de calorías que proporciona energía. Además, el sabor de la bebida cambia, volviéndose más apetitoso, lo cual propicia su ingesta (Martínez et al., 2006).

Es importante mencionar que no todos los sabores amargos que son endulzados con azúcar son ingeridos. Galindo y López-Espinoza (2006) reportaron que existen sabores amargos que aun siendo mezclados con una fuente alterna de calorías no son consumidos por los efectos del sabor. Utilizaron a un grupo de ratas que expusieron a sustancias con contenido energético, pero con sabor no aceptado durante un estado de privación. Reportaron que las ratas a pesar del contenido calórico de la sustancia y el estado de necesidad no ingirieron la sustancia. De esta manera concluyeron que el sabor es un factor determinante para el consumo de alimento.

Una particularidad de los resultados obtenidos en el presente estudio fueron las diferencias en las cantidades consumidas de café con cafeína y café sin cafeína. Se registró una marcada relación entre la cantidad de alimento consumida y la dosis de café utilizada, al parecer relacionada con los efectos posingestivos. Le Magnen (1999) afirmó que los efectos posingestivos están asociados con las propiedades de los alimentos. De tal manera que las consecuencias posingestivas pueden mo- 
dificar la preferencia y selección de los alimentos en la dieta de los organismos originando una aversión (efectos posingestivos negativos) o preferencia (efectos posingestivos positivos) a cierto tipo de alimentos (Burritt \& Provenza, 1992)

Por otra parte, el cambio de sabor de la bebida debido a las concentraciones de café utilizadas provocó la disminución en el consumo del líquido y del alimento. Esta característica probablemente propició que al aumentar la dosis de café el consumo de la bebida disminuyera de manera proporcional, es decir, a mayor concentración de café con cafeína o sin cafeína un menor consumo. Un factor de importancia para que se observara este fenómeno fue la secuencia en la que se presentaron las concentraciones de café. Lo anterior es posible observarlo en los sujetos que fueron expuestos de forma ascendente a las concentraciones de café sin cafeína (grupo 1 de machos y hembras), los cuales no presentaron este efecto.

Adicionalmente, el proceso de la regulación energética puede explicar la redistribución del consumo de alimento en relación con la concentración de café utilizada. Al respecto Rothschild (1971) afirmó que un elemento de importancia en el consumo de alimento es la concentración de solutos que permiten a los organismos determinar qué tan apetitoso es. En el caso del café dicha palatabilidad estuvo determinada por la cantidad empleada en las soluciones y el contenido de cafeína de la mezcla, la cual cambió en relación con el café empleado (con cafeína y sin cafeína).

La aproximación de la psicología experimental a los efectos del consumo de café radica en la importancia social y económica que éste tiene en el mundo. Debido a que se considera como un producto de gran consumo debido a sus propiedades estimulantes, que junto con el tabaco y el té son considerados adictivos. Por esto, el consumo de café en los humanos se vuelve un complejo fenómeno de estudio, ya que no se ha determinado si el consumo de esta bebida es debido al café o la cafeína. Además, se ha considerado la existencia de diversos factores que pueden determinar su consumo como: la clase social, los hábitos alimenticios, ubicación geográfica, estados fisiológicos, entre otros. Lo anterior no permite clarificar cuál es el principal factor que determina que el café sea consumido en los seres humanos. Sin embargo, se podría identificar en los animales si es el sabor del café o la concentración de cafeína, proporcionando de forma separada el sabor del café y la cafeína.

A pesar de que en el presente estudio no se separaron estas propiedades, se empleó una bebida que tenía menor concentración de cafeína como lo fue el café descafeinado. Se observó que la ingesta de esta bebida fue mayor en comparación con el café con cafeína, aunque la concentración de café fue la misma en las dos condiciones, la concentración de cafeína no fue similar. Esto puede llevar a discutir que probablemente el sabor del café no es el único factor que delimita la ingesta de la bebida, sino la concentración de cafeína también es un factor de importancia. Lo anterior lleva a sugerir que las bebidas con mayor concentración de cafeína su consumo es menor.

Para finalizar, se considera necesario realizar otros estudios que contribuyan a la explicación del consumo de café en relación con su sabor y los efectos posingestivos de la cafeína, así como los determinantes fisiológicos y psicológicos que delimitan este fenómeno de estudio, pues, como se ha dicho, el estudio del consumo de café es un tema que genera interés.

\section{Referencias}

Bennett, G. \& Galef, J.R. (1981). Development of Flavor Preferente in Man and Animals: The Role of Social and Nonsocial Factors. Development of Perception, 1, 411-431.

Burritt, E.A. \& Provenza, F.D. (1992). Lambs form Preferentes for Nonnutritive Flavors Paired with Glocose. Journal Animal Science, 70, 1133-1136.

Capaldi, E. (1996). Why We Eat, What We Eat: The Psychology of Eating. American Psychological Association. 
Chou, T. (1992). Wake Up and Smell the Coffee: Caffeine, Coffee, and the Medical Consequences. West Journal Medical, 157, 544-553.

Cuadrado- Calleja, P. (2003). Alcoholismo y drogodependencia. Enfermeria, 21 (11), 291-304.

Fedorchak, P., Mesita, J., Plater, S. \& Brougham, K. (2002). Caffeine-Reinforced Conditioned Flavor Preference in Rats. Behavioral Neuroscience, 116 (2), 334-346.

Galindo, A. \& López-Espinoza A. (2006). Efectos del sabor y del contenido calórico del agua sobre la conducta alimentaria durante un periodo de privación de comida en ratas albinas. Revista Mexicana de Análisis de la Conducta, 2, 95-109.

Griffiths, R., Bigelow, G., \& Liebson, I. (1989) Reinforcing Effects of Caffeine in Coffee and Capsules. Journal of the Experimental analysis of Behavior 52, 127-140.

James, J. (1997) Caffeine and Health. Academic Press, San Diego.

Le Magnen, J. (1999). Efficacy of Olfactory, Tactile and other Food Stimuli in the Acquisition and Manifestation of Appetite in Rats. Appetite, 33 (1), 43-51 . Esta versión en inglés fue traducida por Nancy Julien and edited by David A. Booth and Louise Thibault. El original es de 1959. López- Espinoza (2004). El fenómeno alimentario: ¿un problema biológico o psicológico? Cuadernos de nutrición, 27, 173-177.

Martínez, A.G \& López-Espinoza, A. (2007). Efectos post-privación con dos alternativas energéticas en ratas. Revista Mexicana de Análisis de la Conducta 1, 43-60.

Martínez, A.G., López-Espinoza, A. \& Martínez, H. (2006). Efectos de modificar el contenido energético del agua sobre el peso corporal, consumo de agua, alimento y calorías en ratas. Universitas Psicológica, 5 (2), 361-370.
Messer, E. (2002). Perspectivas Antropológicas sobre la dieta. En J. Contreras (comp.). Alimentación y cultura (pp. 85-109). España: Publicaciones Universidad de Barcelona.

Prasad, C. (1998). Food, Mood and Health: A Neurobiologic Outlook. Food and Mood Brazilian Journal of Medical and Biological Research, 31 (12), 1517-1527.

Rothschild, G.H. (1971). Patterns of Concentration Preference for Glucose and Sodium Chloride in Rats. The Psychological Record, 21, 487-496.

Rozin, P. (2002). Perspectivas psicobiológicas sobre las preferencias y aversiones alimentarias. En J. Contreras (comp). Alimentación y cultura (pp. 85-109). España: Publicaciones Universitat de Barcelona.

Sclafani, A. (1990). Nutritional Based Learned Flavour Preferences in Rats. En E. Capaldi y T. Powley (comp). Taste, Experience and Feeding. American Psychological Association.

Stafford, T. (2003). Psychology in the Coffee Shop. The Psychologist, 16, 358-359.

Tieges, Z., Ridderinkhof, R., Snel, J. \& Kok, A. (2004). Caffeine Strengthens Action Monitoring: Evidence from the Error-Related Negativity. Cognitive Brain Research, 21, 87-93.

Uraga, C., Guijarro, C., Pozas, R. \& Blanco, A. (2002). Algunos principios activos del café, del té y del cacao. Spin Cero, 6, 16-19.

Yeomans, M., Jackson, A., Lee, M., Steer, B., Tinley, E., Durlach, P. \& Rogers, P. (2000). Acquisition and Extinction of Flavour Preferences Conditioned by Caffeine in Human. Appetite 35, 131-141.

Yeretzian, P., Pollien, P., Lindinger, C. \& Ali, S. (2004). Individualization of Flavour Preferences: Toward a Consumer-Centric and Individualized Aroma Science. Comprehensive Reviews in food science and food safety, 3, 152-159. 
